\section{Anemia em crianças menores de cinco anos que freqüentam creches públicas do município do Rio de Janeiro, Brasil}

\section{Anemia in children under five years old attended at public day care centers from Rio de Janeiro, Brazil}

Isabela Escórcio Augusto da Matta 1

Gloria Valeria da Veiga 2

Mirian Ribeiro Baião 3

Marta Maria Antonieta de Souza Santos 4

Ronir Raggio Luiz 5

I-4 Instituto de Nutrição Josué de Castro. Centro de Ciências da Saúde. Universidade Federal do Rio de Janeiro. Bloco J, 2. andar. Av. Brigadeiro Trompowisky, s. n., Cidade Universitária. Ilha do Fundão. Rio de Janeiro, RJ, Brasil. CEP: 21.949-900

2 Núcleo de Estudos Saúde Coletiva. Universidade Federal do Rio de Janeiro, RJ, Brasil

\section{Resumo}

Objetivos: estimar a prevalência de anemia em crianças matriculadas em creches municipais do Rio de Janeiro e identificar os subgrupos de maior risco.

Métodos: foram avaliadas 865 crianças. A hemoglobina $(\mathrm{Hb})$ foi dosada em fotômetro portátil (HemoCue) $e$ a anemia foi definida quando $\mathrm{Hb}<11 \mathrm{~g} / \mathrm{dL}$ e $<9,5 \mathrm{~g} / \mathrm{dL}$ para maiores e menores de seis meses, respectivamente. Foram obtidas informações biológicas e socioeconômicas através de questionários aplicados às mães.

Resultados: A prevalência de anemia foi de 47,3\%. As crianças anêmicas apresentaram médias de z escore de peso para a idade $(-0,239)$ e a altura para a idade (-0,548) mais baixas do que as não-anêmicas. $O$ risco de anemia foi maior para as crianças com idade abaixo de dois anos (razão de prevalência $[R P]=1,73$; intervalo de confiança [IC95\%: 1,52-1,97), para as que tinham pais com menos de quatro anos de estudo ( $R P=1,57$; IC95\%:1,24-1,99) e moravam em domicílios com mais de oito moradores ( $R P=1,45$; IC95\%:,1,07-1,95). .

Conclusões: a prevalência de anemia foi elevada. As crianças com mais baixo peso e estatura para a idade, menores de dois anos, que moravam em residências com número elevado de pessoas e que tinham pais com baixa escolaridade foram as mais vulneráveis à anemia, devendo ser alvo de políticas de controle e prevenção.

Palavras-chave Anemia, Creches, Criança 


\section{Introdução}

A prevalência de anemia por carência de ferro vem aumentando nas últimas décadas e acomete cerca de dois bilhões de habitantes em todo o mundo. ${ }^{1}$ Destaca-se como a principal carência nutricional, dada sua magnitude e os efeitos deletérios à saúde do indivíduo. ${ }^{2}$

Crianças menores de cinco anos estão entre os grupos mais vulneráveis à anemia, devido ao aumento das necessidades de ferro imposto pela expansão da massa celular e crescimento dos tecidos. ${ }^{3}$ Maiores prevalências são encontradas em populações infantis de países em desenvolvimento quando comparadas aos desenvolvidos. ${ }^{1}$ Desigualdades no consumo de carne e de alimentos enriquecidos, na disponibilidade de suplementos de ferro, no acesso à assistência médica, na freqüência das infecções e parasitas intestinais contribuem para essas diferenças. 4

As principais consequiências da anemia por falta de ferro em crianças são o déficit no desenvolvimento psicomotor e na função cognitiva, maior suscetibilidade às infecções e redução da força muscular. 3 Vários fatores têm sido investigados na associação com a anemia, entre estes, a prematuridade, 5 o baixo peso de nascimento, 6 o desmame precoce 7 e os erros alimentares. 8 Os fatores envolvidos na etiologia da anemia ocorrem dentro de um contexto político, econômico e social que, quando adverso, levam a uma precariedade da qualidade de vida e saúde da população e colaboram para o agravamento do quadro de carências nutricionais em crianças. 9

No Brasil não existem informações de âmbito nacional sobre a extensão e a distribuição geográfica da prevalência da anemia ferropriva. Com base em resultados de vários estudos locais realizados na última década, a maioria referente à clientela de serviços de saúde, observa-se que as prevalências variam de $36,4 \%$ na Paraíba ${ }^{10}$ a $73,2 \%$ em quatro municípios da zona da Mata Meridional de Pernambuco. ${ }^{11}$ Dados de aumento da prevalência advém da cidade de São Paulo, a qual passou de 35,6\% entre 1984 e 1985 para 46,9\% em 1995 e 1996, em crianças menores de cinco anos de idade. 12

Esses dados indicam que a anemia constitui um problema nutricional de grande magnitude em nosso meio, superando a desnutrição energético-protéica, que vem declinando nas últimas décadas. ${ }^{13}$ Diante da extensão do problema, dos danos que pode causar à saúde da criança e da viabilidade de controle, pode-se considerar que a anemia é um problema de saúde pública o qual demanda maiores investi- gações.

No Brasil, entre 10 a $15 \%$ dos pré-escolares freqüentam creches gratuitas e a demanda por esses serviços tende a aumentar com a participação da mulher no mercado de trabalho. ${ }^{14}$ Em crianças assistidas em creches foram encontradas prevalências de até $81 \%$ em Recife 15 e bem mais baixas $(15,4 \%)$ no Rio de Janeiro. ${ }^{16}$ Entretanto, o estudo nesta cidade abrangeu apenas crianças matriculadas em duas creches públicas.

O presente estudo tem como objetivo avaliar a prevalência de anemia em crianças assistidas em nove creches que fazem parte do Programa Rio Creche da Secretaria Municipal de Desenvolvimento Social (SMDS) do Rio de Janeiro e identificar subgrupos mais vulneráveis ao problema. As creches estão localizadas em comunidades atendidas pelo Projeto Favela-Bairro, consideradas áreas de pobreza e alvo de intervenções físicas de transformação ambiental e social, originadas de ações públicas articuladas.17 Os resultados poderão subsidiar a implementação de intervenções voltadas para a prevenção e o controle do problema.

\section{Método}

O estudo foi do tipo transversal, realizado com 865 crianças menores de cinco anos de idade que frequientavam nove das 29 creches que compunham o Programa Rio Creche da SMDS do município do Rio de Janeiro no estado do Rio de Janeiro, Brasil, o qual atendia cerca de 3080 crianças matriculadas no ano de 2000. O critério de amostragem considerou prevalência de anemia de 57,6\%,5 com erro tolerável de $2,5 \%$ e significância de $5 \%$, perfazendo um total de 980 crianças. Prevendo-se perda de $25 \%$, com base em estudo piloto realizado em uma das creches municipais, estimou-se número inicial de 1225 crianças. Foram selecionadas, por conveniência, nove creches nas quais estavam matriculadas 1217 crianças, com o cuidado de ter representadas as cinco Coordenadorias Regionais do município. Houve perda de 328 crianças $(27,0 \%)$ em decorrência de ausência no dia da coleta de dados $(n=238)$ ou não-autorização para participação no estudo por parte dos responsáveis $(n=90)$, totalizando 889 crianças avaliadas. Dessas, 24 (14 do sexo masculino e 10 do sexo feminino) foram excluídas por apresentarem dados incompletos, resultando 865 crianças, com perda total de $29 \%$. Para 248 crianças não houve resposta ao questionário que avaliou as variáveis socioeconômicas e biológicas.

$\mathrm{O}$ critério de amostragem por conveniência se 
deu em função da dificuldade de acesso às creches localizadas em regiões de alto índice de violência, o que poderia inviabilizar a realização do estudo. Essa limitação em função da não-aleatoriedade do critério de seleção da amostra, pode ser minimizada pelo fato de a matrícula das crianças estar condicionada a critérios gerais, padronizados pela SMDS, o que faz supor uma população, relativamente, homogênea.

A coleta de dados foi efetuada pelos pesquisadores, devidamente treinados, no período de outubro de 2000 a dezembro de 2001.

A avaliação da anemia foi realizada através da dosagem de hemoglobina $(\mathrm{Hb})$, utilizando-se fotômetro portátil (HemoCue) para leitura direta em amostras sangüíneas obtidas por puntura do pé ou do dedo com lancetas descartáveis. Esse equipamento foi validado e tem sido recomendado em investigações populacionais sobre a prevalência de anemia devido à utilização de pequeno volume sangüíneo $(10 \mu 1)$ e à imediata obtenção do resultado.18 Foram consideradas anêmicas as crianças com seis ou mais meses de idade com $\mathrm{Hb}<11 \mathrm{~g} / \mathrm{dL}$ e as menores de seis meses com $\mathrm{Hb}<9,5 \mathrm{~g} / \mathrm{dL}$, segundo a World Health Organization (WHO). 19

A avaliação nutricional foi realizada por meio de medidas antropométricas como peso e estatura, seguindo técnica de Gordon et al.,20 com as crianças descalças e usando o mínimo de vestimentas. O peso foi medido em balança eletrônica, com capacidade de $150 \mathrm{Kg}$, com variação de $50 \mathrm{~g}$ para as maiores de dois anos e balança eletrônica pediátrica (tipo pesa bebê), com capacidade de $15 \mathrm{Kg}$ e variação de $10 \mathrm{~g}$, para as menores de dois anos. A altura de maiores de dois anos, foi obtida com antropômetro portátil, estando a criança em posição ortostática. Para menores de dois anos, o comprimento foi aferido com um antropômetro de madeira, com amplitude de 0 a $100 \mathrm{~cm}$, estando as crianças em posição horizontal. Ambos os instrumentos apresentavam variação de $0,1 \mathrm{~cm}$ e as medidas foram aferidas duas vezes, tirando-se a média, admitindo-se uma variação máxima de $0,5 \mathrm{~cm}$ entre as duas. Caso a variação excedesse a esse valor, ambas eram repetidas. Avaliaram-se os índices peso para idade (P/I), altura para idade $(\mathrm{A} \backslash \mathrm{I})$ e peso para altura $(\mathrm{P} / \mathrm{A})$, adotandose $-2 \mathrm{z}$ escore como ponto de corte para definir déficits pôndero-estaturais $\mathrm{e}+2 \mathrm{z}$ escore de P/A para definir sobrepeso, com base na referência do National Center for Health and Statistics, segundo a WHO. 21

As informações sobre peso ao nascer, tempo de gestação, exposição ao aleitamento materno, idade materna, escolaridade dos pais, renda familiar per capita, número de moradores na família e condições de moradia foram obtidas através de questionário auto-preenchido pelos responsáveis, após explicação prévia. A data de nascimento para avaliação da idade foi obtida através da ficha de matrícula da criança na creche. Esses dados foram transformados em variáveis e categorizados para avaliação dos subgrupos de maior risco para anemia.

A análise estatística envolveu a estimativa da prevalência de anemia e seus respectivos intervalos de confiança 95\% (IC95\%). As comparações entre os grupos de crianças anêmicas e não-anêmicas foram feitas através do teste $t$ de Student para as médias da variável contínua (z escore dos índices antropométricos) e o teste Qui-Quadrado para as freqüências das variáveis categóricas, sendo consideradas diferenças significativas quando o valor de $p$ foi $<0,05$. Através da avaliação da associação das diversas variáveis com a prevalência de anemia foram identificados os subgrupos de maior risco, calculando-se a razão de prevalências (RP) e seus respectivos intervalos de confiança a $95 \%$. Considerando o reconhecido efeito da idade na anemia,7,22-23 será realizada uma análise controlada para essa variável, por regressão logística, apenas para aquelas covariáveis que se mostrarem estatisticamente significativas na análise univariada.

Todas as análises foram realizadas através do programa Epi-info, versão 6.04 .

O estudo teve aprovação do comitê de ética do Hospital Municipal Souza Aguiar do Rio de Janeiro e as autorizações por escrito (Termo de Consentimento Esclarecido) foram obtidas dos dirigentes das creches e dos responsáveis pelas crianças em conformidade com os princípios éticos recomendados pela Resolução 196/96 do Conselho Nacional de Saúde.

\section{Resultados}

A comparação entre as 617 crianças cujos questionários foram devolvidos e as 248 para as quais não se obteve resposta, não mostrou diferença significativa quanto às variáveis antropométricas e sexo, mas havia maior $(p<0,05)$ proporção de crianças abaixo de 24 meses $(29,8 \%$ vs $20,9 \%)$ e de crianças anêmicas $(54,0 \%$ vs $44,7 \%)$ entre as que não retornaram o questionário.

A prevalência de anemia foi de 47,3\% ( $n=409)$. A maioria das crianças $(76,5 \%, \mathrm{n}=662)$ tinha idade acima de 24 meses, 50,9\% $(n=440)$ eram do sexo masculino e $49,1 \% \quad(n=425)$ do sexo feminino. Grande parte das crianças $(89,2 \% \mathrm{n}=518)$ nasceu a termo e $9,2 \%(n=53)$ apresentaram baixo peso ao 
nascer. Quanto ao aleitamento materno, 95,0\% $(n=583)$ foi amamentada ao peito, sendo que $73,8 \%$ $(n=447)$ receberam leite materno exclusivo em algum momento da vida. A prevalência de déficits de P/A, P/I e A/I foi de 0,8\% ( $n=7), 2,7 \%(n=23)$, e $6,0 \%(n=52)$, respectivamente e de sobrepeso foi de $3,8 \%(\mathrm{n}=33)$, com base em P/A. A maioria das mães $(93,8 \%, n=546)$ tinha vinte anos de idade ou mais (Tabela 1).

As médias de $\mathrm{z}$ escore dos índices antro- pométricos foram mais baixas para as crianças anêmicas, porém com significância estatística apenas para P/I e A/I (Tabela 2).

Em relação à renda, $89 \%$ das famílias das crianças $(n=433)$ viviam com menos de um SMPC, enquanto que $91,5 \%(n=463)$ das mães e $88,2 \%$ $(\mathrm{n}=366)$ dos pais apresentaram quatro ou mais anos de estudo (Tabela 3). Mais de 90\% das famílias apresentaram condições satisfatórias de moradia quanto a presença de água encanada $(n=552)$ e de banheiro

Tabela 1

Freqüência de anemia em relação a características biológicas e demográficas, razão de prevalência e intervalo de confiança para $95 \%$ (195\%) em crianças assistidas em nove creches do programa Favela-Bairro. Rio de Janeiro (capital), 2000 a 2001.

\begin{tabular}{|c|c|c|c|c|c|c|}
\hline \multirow[t]{2}{*}{ Variáveis } & \multicolumn{6}{|c|}{ Freqüência de anemia } \\
\hline & $\mathrm{n}$ & $\mathrm{n}$ & $\%$ & $\mathrm{RP}$ & IC95\% & $\mathrm{p}$ \\
\hline Idade & 865 & & & & & \\
\hline$=24,0$ meses & 662 & 267 & 40,3 & 1,00 & & \\
\hline$<24,0$ meses & 203 & 142 & 70,0 & 1,75 & $1,52-1,97$ & 0,001 \\
\hline Sexo & 865 & & & & & \\
\hline Feminino & 425 & 199 & 46,8 & 1,00 & & \\
\hline Masculino & 440 & 210 & 47,7 & 1,02 & $0,89-1,17$ & 0,79 \\
\hline Peso ao nascer & 579 & & & & & \\
\hline$=2500$ gramas & 526 & 231 & 43,9 & 1,00 & & \\
\hline$<2500$ gramas & 53 & 25 & 47,2 & 1,07 & $0,80-1,45$ & 0,65 \\
\hline Tempo de gestação & 581 & & & & & \\
\hline Termo & 518 & 233 & 45,0 & 1,00 & & \\
\hline Pré-termo & 63 & 25 & 39,7 & 0,88 & $0,64-1,21$ & 0,42 \\
\hline Aleitamento materno & 614 & & & & & \\
\hline Sim & 583 & 264 & 45,3 & 1,00 & & \\
\hline Não & 31 & 11 & 35,5 & 1,28 & $0,79-2,07$ & 0,29 \\
\hline Aleitamento materno exclusivo & 606 & & & & & \\
\hline Sim & 447 & 202 & 45,2 & 1,00 & & \\
\hline Não & 159 & 67 & 42,1 & 1,07 & $0,87-1,32$ & 0,51 \\
\hline \multicolumn{7}{|l|}{ Estado nutricional } \\
\hline Altura/ Idade & 865 & & & & & \\
\hline$=-2 \mathrm{z}$ escore & 813 & 378 & 46,5 & 1,00 & & \\
\hline$<-2$ z escore & 52 & 31 & 59,6 & 1,28 & $1,01-1,62$ & 0,07 \\
\hline Peso/ Altura & 865 & & & & & \\
\hline$=2 \mathrm{z}$ escore & 832 & 397 & 47,7 & 1,00 & & \\
\hline$>2 \mathrm{z}$ escore & 33 & 12 & 36,4 & 0,76 & $0,48-1,20$ & 0,20 \\
\hline Peso/ Altura & 865 & & & & & \\
\hline$=-2 \mathrm{z}$ escore & 858 & 405 & 47,2 & 1,00 & & \\
\hline$<-2$ z escore & 7 & 4 & 57,1 & 1,21 & $0,63-2,31$ & 0,60 \\
\hline Peso/ Idade & 865 & & & & & \\
\hline$=-2 \mathrm{z}$ escore & 842 & 395 & 46,9 & 1,00 & & \\
\hline$<-2$ z escore & 23 & 14 & 60,9 & 1,30 & $0,93-1,81$ & 0,19 \\
\hline Idade da mãe & 582 & & & & & \\
\hline$=20$ anos & 546 & 241 & 44,1 & 1,00 & & \\
\hline$<20$ anos & 36 & 19 & 52,8 & 1,20 & $0,87-1,65$ & 0,31 \\
\hline
\end{tabular}


Médias e desvio padrão de z escore para os índices antropométricos em crianças anêmicas e não-anêmicas assistidas em nove creches do programa Favela-Bairro. Rio de Janeiro (capital), 2000 a 2001.

\begin{tabular}{|c|c|c|c|c|c|c|c|}
\hline \multirow[b]{2}{*}{ Índices } & \multicolumn{3}{|c|}{$\begin{array}{c}\text { Anêmicos } \\
(H b<11,0 \mathrm{~g} / \mathrm{dL}) \\
(\mathrm{n}=409)\end{array}$} & \multicolumn{3}{|c|}{$\begin{array}{c}\text { Não-anêmicos } \\
(\mathrm{Hb} \geqslant 11,0 \mathrm{~g} / \mathrm{dL}) \\
(\mathrm{n}=456)\end{array}$} & \multirow[b]{2}{*}{$p$} \\
\hline & $\overline{\mathbf{x}}$ & \pm & $d p$ & $\overline{\mathbf{x}}$ & \pm & $d p$ & \\
\hline Peso / Idade & $-0,239$ & \pm & 1,095 & 0,038 & \pm & 1,216 & 0,001 \\
\hline Peso / Altura & 0,200 & \pm & 0,860 & 0,319 & \pm & 0,928 & 0,07 \\
\hline Altura / Idade & $-0,548$ & \pm & 1,203 & $-0,237$ & \pm & 1,237 & 0,001 \\
\hline
\end{tabular}

$(\mathrm{n}=584)$, porém menos de $50 \%$ tinham condições adequadas de coleta de lixo $(n=282)$ e de escoamento de esgoto $(n=211)$ (Tabela 4$)$.

A faixa etária das crianças, a educação paterna e o número de pessoas residentes no domicílio mostraram uma associação significativa com a anemia (Tabela 1 e 3). As crianças com menos de 24 meses de idade apresentaram prevalência de anemia $75 \%$ maior do que as com mais de 24 meses $(\mathrm{RP}=1,75$; IC95\%: 1,52-1,97). A escolaridade paterna abaixo de quatro anos aumentou a prevalência de anemia em 57\% (RP=1,57; IC95\%: 1,24-1,99) e os domicílios com mais de oito pessoas em $45 \%$ $(\mathrm{RP}=1,45$; IC95\%: 1,07-1,95). A associação com a escolaridade paterna e o número de pessoas na família foi mantida após ajuste para o efeito da idade (Odds ratio bruto e ajustado foi 2,65 e 2,82 para escolaridade do pai e 2,06 e 2,08 para número de pessoas na família, tendo como referência menos de quatro pessoas).

As variáveis referentes às condições de moradia não mostraram associação com anemia (Tabela 4), assim como as demais variáveis avaliadas (Tabela $1 \mathrm{e}$ $3)$.

\section{Tabela 3}

Freqüência de anemia em relação às condições socioeconômicas, razão de prevalência e intervalo de confiança para $95 \%$ (IC95\%) em crianças assistidas em nove creches do programa Favela-Bairro. Rio de Janeiro (capital), 2000 a 2001.

\begin{tabular}{|c|c|c|c|c|c|c|}
\hline \multirow[t]{2}{*}{ Variáveis } & \multicolumn{6}{|c|}{ Freqüência de Anemia } \\
\hline & $\mathrm{n}$ & $\mathrm{n}$ & $\%$ & $\mathrm{RP}$ & IC95\% & $p$ \\
\hline Renda familiar per capita (sm) & 487 & & & & & \\
\hline$<0,50$ & 228 & 110 & 48,2 & 1,24 & $0,87-1,78$ & 0,22 \\
\hline$=0,50<1,00$ & 205 & 82 & 40,0 & 1,03 & $0,71-1,50$ & 0,88 \\
\hline$=1,00$ & 54 & 21 & 38,9 & 1,00 & & \\
\hline Número de pessoas na família & 594 & & & & & \\
\hline$<4$ pessoas & 140 & 56 & 40,0 & 1,00 & & \\
\hline$=4<8$ pessoas & 397 & 175 & 44,1 & 1,10 & $0,87-1,39$ & 0,40 \\
\hline$=8$ pessoas & 57 & 33 & 57,9 & 1,45 & $1,07-1,95$ & 0,02 \\
\hline Educação materna & 506 & & & & & \\
\hline$=4$ anos & 463 & 204 & 44,1 & 1,0 & & \\
\hline$<4$ anos & 43 & 23 & 53,5 & 1,21 & $0,90-1,63$ & 0,23 \\
\hline Educação paterna & 415 & & & & & \\
\hline$=4$ anos & 366 & 152 & 41,5 & 1,0 & & \\
\hline$<4$ anos & 49 & 32 & 65,3 & 1,57 & $1,24-1,99$ & 0,001 \\
\hline
\end{tabular}

$\mathrm{SM}=$ salário mínimo 
Tabela 4

Freqüência de anemia em relação às condições de moradia, razão de prevalência e intervalo de confiança para 95\% (IC95) em crianças assistidas em nove creches do programa Favela-Bairro. Rio de Janeiro (capital); 2000 a 2001.

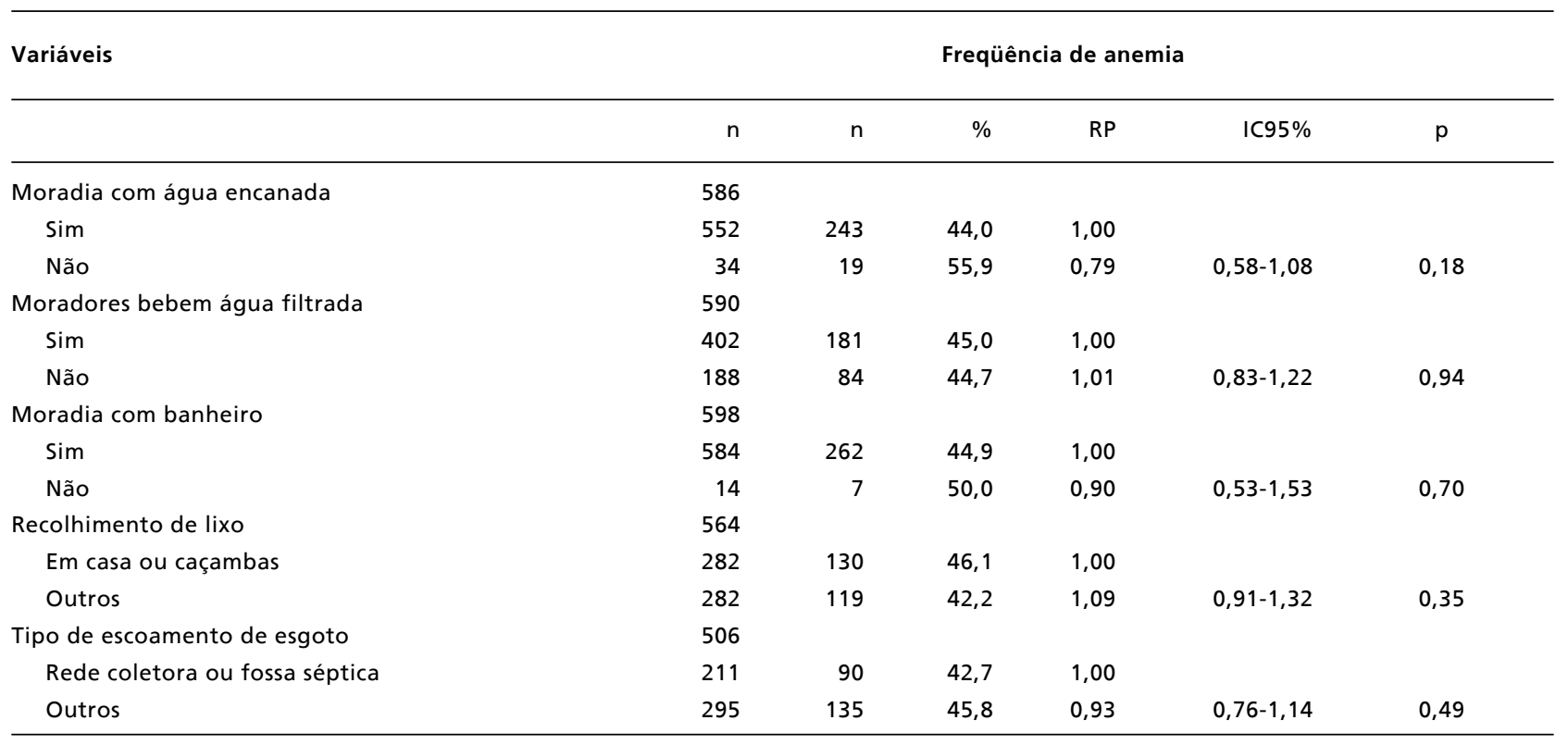

\section{Discussão}

A prevalência de anemia ferropriva observada no presente estudo $(47,3 \%)$ foi bem maior do que a encontrada em um estudo anterior, realizado em creches públicas do Rio de Janeiro, 16 sendo classificada como alta prevalência, segundo o critério da WHO.1 Prevalência menor $(28,6 \%)$ também foi observada em creches na cidade de Vitória, Espírito Santo. 24 Valor semelhante $(47,8 \%)$ foi relatado por Silva et al.,22 em creches de Porto Alegre. Prevalências mais elevadas foram encontradas em creches de São Paulo $(62,5 \%)^{25}$ e em Cuiabá $(63,0 \%) .^{23}$

A importância das creches na proteção da saúde das crianças tem sido alvo de controvérsias. Collet et al.26 e Fonseca et al.27 apontam que crianças que freqüentam creches apresentam maior número de episódios de doenças infecto-contagiosas, com possível associação com desnutrição nas menores de 24 meses. Por outro lado, Silva et al.,28 quando compararam crianças que freqüentavam creches com as que não freqüentavam, dentro de uma mesma comunidade, verificaram melhor estado nutricional entre aquelas que frequientavam as creches há mais de um ano.

Os resultados obtidos no presente estudo reforçam o fato de que crianças com menos de 24 meses são mais suscetíveis à anemia ferropriva, conforme relatado por vários outros autores.7,22-23 Provavelmente, esse fato se deve a alguns aspectos, tais como a maior velocidade de crescimento nessa faixa etária, ao desmame precoce com atraso na introdução de alimentos ricos em ferro na dieta da criança e a maior prevalência de doenças infecciosas nos primeiros anos de vida. 22

No presente estudo não foi observada diferença entre os sexos quanto à prevalência da anemia, semelhante ao observado por Almeida et al. $25 \mathrm{e}$ Neuman et al. ${ }^{7}$ Entretanto, no Estado da Paraíba, Oliveira et al. ${ }^{29}$ encontraram maior risco para préescolares do sexo masculino, o que também foi relatado por Alberico et al. ${ }^{5}$ no Rio de Janeiro e por Torres et al. 30 em São Paulo.

O baixo peso ao nascer é tido como um dos fatores pré-disponentes mais importantes na gênese da anemia ferropriva em lactentes, ${ }^{2} 2$ seja pela desnutrição intra-uterina ou pela prematuridade, que resultam na redução das reservas de ferro acumuladas durante a gestação. 30 Piscoya11 avaliou lactentes aos 12 meses, em quatro municípios de Pernambuco, e encontrou associação de baixo peso ao nascer com a anemia. Uchimura et al. 31 relataram que crianças que nascem com baixo peso têm uma probabilidade triplicada de serem anêmicas. 
Entretanto, no presente estudo, essa associação não foi observada, semelhante ao encontrado por outros autores. 5,7

Esperava-se que crianças que receberam leite materno apresentassem menor prevalência de anemia do que as que nunca foram amamentadas. Todavia, o aleitamento materno não mostrou associação com a anemia, o que também foi observado por outros autores. 7,22,32 No presente estudo houve dificuldade de se obter informação quanto à duração do aleitamento materno, o que consiste em uma limitação para a interpretação da associação da anemia com essa variável. Porém, mesmo quando essa duração foi avaliada, a associação também não foi observada. $8,11,22$

A anemia é uma carência nutricional que pode ocorrer independente da desnutrição, apesar de haver tendência dessas duas doenças estarem associadas. 7,30 Neste estudo, crianças mais baixas e mais magras parecem apresentar maior risco de anemia, pois, embora não tenha sido encontrada associação entre desnutrição ( $<-2 \mathrm{z}$ escore) e anemia, semelhante a outros estudos, 5,25 observaram-se médias mais baixas de $\mathrm{z}$ escore dos índices P/I e A/I entre as crianças anêmicas.

A renda familiar per capita não mostrou associação significativa com a anemia, em concordância com alguns estudos25,32,33 e diferindo de outros, 7,11,12,22 que mostraram maior risco para crianças mais pobres. A não-associação com a renda, neste estudo, pode estar relacionada à homogeneidade da amostra, considerando que $89 \%$ das famílias viviam com menos de um salário mínimo per capita. O mesmo pode-se supor em relação a não-associação observada com as condições de moradia.

A maior prevalência de anemia encontrada em filhos de pais com menor escolaridade também foi observada em outros estudos, 7,34 assim como em crianças residentes em domicílios com maior número de pessoas. ${ }^{7} \mathrm{O}$ maior nível de instrução, provavelmente, pode repercutir em maior chance de trabalho e de renda, condicionando um melhor acesso aos alimentos e cuidados com a saúde da criança. $^{2}$

Algumas limitações podem ser apontadas neste trabalho. Uma delas diz respeito à ausência de informação no cadastro das instituições quanto ao tempo em que a criança freqüentava a creche, não podendo ser investigada a associação com a anemia. O elevado percentual de absenteísmo no dia da coleta de dados e a dificuldade de acesso aos responsáveis pelas crianças geraram perdas além do previsto. Considerando que a perda foi diferencial para as crianças com questionários devolvidos e sem devolução, no que se refere à prevalência de anemia e faixa etária, questiona-se se as associações de anemia com as variáveis investigadas poderiam estar subestimadas.

Apesar das limitações, a importância do presente estudo incide no fato de identificar, dentro de uma população relativamente homogênea, subgrupos mais vulneráveis para o desenvolvimento da anemia, com destaque para as crianças menores de dois anos de idade. Ressalta-se que o percentual de crianças matriculadas nessa faixa etária nas creches foi mais baixo que o de crianças maiores, semelhante ao encontrado por Fisberg et al. 6 Nesse sentido, o subgrupo mais vulnerável à anemia parece não estar suficientemente assistido pelos programas de creches públicas, corroborando o ponto de vista de Silva et al. 28

Os presentes resultados alertam para a necessidade de implantação e reforço de medidas voltadas, principalmente, para os subgrupos de maior risco, visando a prevenção, controle e tratamento da anemia ferropriva. Medidas como suplementação de ferro aliada à orientação alimentar, campanhas de promoção do aleitamento materno exclusivo até os seis meses de idade e a fiscalização da fortificação de alimentos com ferro podem ser bastante eficazes para a obtenção de êxito.

Crianças menores de dois anos, assim como aquelas cujos pais apresentam baixa escolaridade e as que pertencem a famílias numerosas devem ser priorizadas para assistência em creches públicas, nas quais a alimentação saudável deve estar garantida como medida fundamental para o enfrentamento da anemia ferropriva.

\section{Agradecimentos}

À Fundação Universitária José Bonifácio pela concessão de auxílio financeiro para realização deste estudo e a nutricionista Ana Cristina de Campos Rocha pelo auxílio na coleta de dados. 


\section{Referências}

1. WHO (World Health Organization). Iron deficiency anemia: assessment, prevention and control. A guide for programme managers. Geneva; 2001.

2. Osório MM. Fatores determinantes da anemia em crianças. J Pediatr [Rio J] 2002; 78: 269-78.

3. Olivares MG, Walter TK. Consecuencias de la deficiencia de hierro. Rev Chil Nutr 2003; 30: 226-33.

4. Lönnerdal B, Dewey KG. Epidemiologia da deficiência de ferro no lactente e na criança: deficiência de ferro no lactente e na criança. Anais Nestlé 1996; 52: 11-7.

5. Alberico APM, Veiga GV, Baião MR, Santos MMAS, Souza SB, Szarfac SC. Iron deficiency anaemia in infants attended at municipal primary health care centres in Rio de Janeiro, Brazil. Nutr Food Sci 2003; 33: 50-5.

6. Fisberg RM, Marchioni DML, Cardoso MRA. Estado nutricional e fatores associados ao déficit de crescimento de crianças freqüentadoras de creches públicas do Município de São Paulo, Brasil. Cad Saúde Pública 2004; 20: 812-7.

7. Neuman NA, Tanaka OY, Szarfarc SC, Guimarães PRV, Victora CG. Prevalência e fatores de risco para anemia no Sul do Brasil. Rev Saúde Pública 2000; 34: 56-63.

8. Alberico APM, Veiga GV, Baião MR, Santos MMAS, Souza SB, Szarfac SC. Breast-feeding, weaning diet and iron deficiency anaemia in infants. Nutr Food Sci 2003; 33: 111-9.

9. Alberico APM. Prevalência de anemia no primeiro ano de vida em crianças atendidas em dois centros de saúde do Município do Rio de Janeiro: associação com alguns fatores de risco, com aleitamento materno e dieta de desmame [dissertação mestrado]. Rio de Janeiro: Instituto de Nutrição da Universidade Federal do Rio de Janeiro; 1999.

10. Oliveira RS, Diniz AS, Benigna MJC, Miranda-Silva SM, Lola MM, Golçalves MC, Asciutti-Moura L, Rivera MA, Santos LMP. Magnitude, distribuição espacial e tendência da anemia em pré-escolares da Paraíba. Rev Saúde Pública 2002; 36: 26-32.

11. Piscoya MDBDV. Anemia ferropriva em crianças aos 12 meses em quatro municípios de Pernambuco [dissertação mestrado]. Recife: Departamento Materno Infantil da Faculdade de Medicina da Universidade Federal de Pernambuco; 2001.

12. Monteiro CA, Szarfarc SC, Mondini L. Tendência secular da anemia na infância na cidade de São Paulo (1984-1996). Rev Saúde Pública 2000; 34: 62-72

13. Monteiro CA, Iunes RF, Gouveia NC, Cardoso MAA. Evolução da desnutrição infantil. In: Monteiro, CA, editor. Velhos e novos males da saúde no Brasil. A evolução do país e de suas doenças. 2. ed. São Paulo: HUCITEC; 2000. p. 93-114.

14. Barros AJD, Gonçalves EV, Borba CRS, Lorenzatto CS, Motta DB, Silva VRL, Schiroky VM. Perfil das creches de uma cidade de porte médio do Sul do Brasil: operação, cuidados, estrutura física e segurança. Cad Saúde Pública 1999; 15: 597-604.

15. Perez JLP, Gonçalvez BPB, Figueiroa FV, Barreto LL, Medeiros JJA, Perez EP, Rocha JA, Brasileiro MC, Silva MMM, Moreira Z. Anemia em crianças menores de três anos: estudo em creches do Recife, PE. Rev IMIP 1998; 12: 19-24.

16. Cople CDS. Sobrecrescimento bacteriano no intestino delgado, absorção e tolerância à lactose e deficiência de ferro em pré-escolares de duas creches do Rio de Janeiro [tese doutorado]. São Paulo: Escola Paulista de Medicina da Universidade Federal de São Paulo; 2001.

17. Azevedo AMF, Engstron EM, Castro IRR, Silva CS, Anjos LA. Saúde e nutrição em escolares. Saúde Foco 1999; 7: 27-32.

18. Hudson-Thomas M, Bingham KC, Simmons WK. An evaluation of the HemoCue for measuring hemoglobin in field studies in Jamaica. Bul World Health Org 1994; 72: 423 26.

19. WHO (World Health Organization). The clinical use of blood in medicine, obstetrics, paediatrics, surgery \& anaesthesia, trauma \& aburns. Geneve; 2001.

20. Gordon CC, Chumlea WC, Roche AF. Stature, recumbent lenght, and weight. In: Lohman TG, Roche AF, Martorell R, editors. Anthropometric standardization reference manual. Champaign: Human Kinetics Books; 1988. p. 3-8.

21. WHO (World Health Organization). Physical status: the use and interpretation of antropometry. Geneva; 1995. (Technical Report Series, 854)

22. Silva LSM, Giugliani ERJ, Aerts DRGC. Prevalência e determinantes de anemia em crianças de Porto Alegre, RS, Brasil. Rev Saúde Pública 2001; 35: 66-73.

23. Brunken GS, Guimarães LV, Fisberg M. Anemia em crianças menores de três anos que freqüentavam creches públicas em período integral. J Pediatr [Rio J] 2002; 78: 506.

24. Almeida ADPC. Prevalência da anemia em crianças de seis meses a seis anos na cidade de Vitória, ES [dissertação mestrado]. Minas Gerais: Departamento de Pediatria da Faculdade de Medicina da Universidade Federal de Minas Gerais; 2000.

25. Almeida CAN, Ricco RG, Ciampo LAD, Souza AM, Pinho AP, Dutra-de-Oliveira JE. Fatores associados a anemia por deficiência de ferro em crianças pré-escolares brasileiras. J Pediatr [Rio J] 2004; 80: 229-34.

26. Collet JP, Ducruet T, Floret D, Cogan-Collet J, Honneger D, Boissel JP. Daycare attendance and risk of first infectious disease. Eur J Pediatr 1991; 150: 214-6.

27. Fonseca W, Kirkwood BR, Barros AJD, Misago C, Correia LL, Flores JA.M, Fuchs SC, Victora CG. Attendance at day care centers increases the risk of childhood pneumonia among the urban poor in Fortaleza, Brasil. Cad Saúde Pública 1996; 12: 133-40.

28. Silva MV, Ometto AMH, Furtuoso COM, Pipitone MAP, Sturion, GL. Acesso à creche e estado nutricional das crianças brasileiras: diferenças regionais, por faixa etária e classe de renda. Rev Nutr 2000; 13: 193-9.

29. Oliveira RS, Diniz AS, Benigna MJC, Miranda-Silva SM, Lola MM, Golçalves MC, Asciutti-Moura L, Rivera MA, Santos LMP. Magnitude, distribuição espacial e tendência da anemia em pré-escolares da Paraíba. Rev Saúde Pública 2002; 36: 26-32. 
30. Torres MAA, Sato K, Juliano Y, Queiroz SS. Terapêutica com doses profiláticas de sulfato ferroso como medida de intervenção no combate à carência de ferro em crianças atendidas em unidades básicas de saúde. Rev Saúde Pública 1994; 26 : 410-5.

31. Uchimura TT, Szarfarc SC, Latorre MRDO, Uchimura NS, Souza SB. Anemia e peso ao nascer. Rev Saúde Pública 2003; 37: 397-403.

32. Rodrigues CRM, Motta SS, Cordeiro AA, Lacerda EMA, Reichenheim ME. Prevalência de anemia ferropriva e marcadores de risco associados em crianças entre 12 e 18 meses de idade atendidas nos Ambulatórios do Instituto de Puericultura e Pediatria Martagão Gesteira. J Pediatr [Rio J] 1997; 73: 189-94.
33. Assis AMO, Santos LMP, Martins MC, Araújo MPN, Amorim DQ, Morris SS, Barreto ML. Distribuição da anemia em pré-escolares do semi-árido da Bahia. Cad Saúde Pública 1997; 13: 237-43.

34. Silva DG, Franceschini SCC, Priore SE, Ribeiro SMR, Szarfarc SC, Souza SB, Almeida LP, Lima NMM, Maffia UCC. Anemia ferropriva em crianças de 6 a 12 meses atendidas na rede pública de saúde do município de Viçosa, Minas Gerais. Rev Nutr 2002; 15: 301-8.

Recebido em 6 de janerio de 2005

Versão final em 4 de maio de 2005

Aprovado em 17 de agosto de 2005 